\title{
Exact Solutions of Nonlinear Partial Differential Equations by the Method of Group Foliation Reduction ${ }^{\star}$
}

\author{
Stephen C. ANCO ${ }^{\dagger}$, Sajid $A L I^{\ddagger}$ and Thomas WOLF ${ }^{\dagger}$ \\ $\dagger$ Department of Mathematics, Brock University, St. Catharines, ON L2S 3 A1 Canada \\ E-mail: sanco@brocku.ca,twolf@brocku.ca \\ $¥$ School of Electrical Engineering and Computer Sciences, \\ National University of Sciences and Technology, H-12 Campus, Islamabad 44000, Pakistan \\ E-mail: sajid_ali@mail.com
}

Received March 05, 2011, in final form July 03, 2011; Published online July 12, 2011

doi:10.3842/SIGMA.2011.066

\begin{abstract}
A novel symmetry method for finding exact solutions to nonlinear PDEs is illustrated by applying it to a class of semilinear reaction-diffusion equations. The method uses a separation ansatz to solve an equivalent first-order group foliation system whose independent and dependent variables respectively consist of the invariants and differential invariants of a given one-dimensional group of point symmetries for the class of reactiondiffusion equations. With this group-foliation reduction method, solutions of the reactiondiffusion equations are obtained in an explicit form, including group-invariant similarity solutions and travelling-wave solutions, as well as dynamically interesting solutions that are not invariant under any of the point symmetries admitted by the equations in this class.
\end{abstract}

Key words: semilinear heat equation; similarity reduction; exact solutions; group foliation; symmetry

2010 Mathematics Subject Classification: 35K58; 35C06; 35A25; 58J70; 34C14

\section{Introduction}

The construction of group foliations using admitted point symmetry groups for nonlinear partial differential equations (PDEs) is originally due to Lie and Vessiot and was revived in its modern form by Ovsiannikov [1]. In general a group foliation converts a given nonlinear PDE into an equivalent first-order PDE system, called the group-resolving equations, whose independent and dependent variables respectively consist of the invariants and differential invariants of a given one-dimensional group of point symmetry transformations. Each solution of the group-resolving equations geometrically corresponds to an explicit one-parameter family of exact solutions of the original nonlinear PDE, such that the family is closed under the given one-dimensional symmetry group acting in the solution space of the PDE.

Because a group foliation contains all solutions of the given nonlinear PDE, ansatzes or differential-algebraic constraints must be used to reduce the group-resolving equations into an overdetermined system for the purpose of obtaining explicit solutions. Compared with classical symmetry reduction $[2,3]$, a main difficulty to-date has been how to find effective, systematic ansatzes that lead to useful reductions.

An important step toward overcoming this difficulty has been taken in recent work $[4,5]$ on finding exact solutions to semilinear wave equations and heat equations with power nonlineari-

\footnotetext{
*This paper is a contribution to the Special Issue "Symmetry, Separation, Super-integrability and Special Functions $\left(\mathrm{S}^{4}\right)$ ". The full collection is available at http://www.emis.de/journals/SIGMA/S4.html
} 
ties. Specifically, this work demonstrates that the group-resolving equations for such nonlinear PDEs have solutions arising from a simple separation ansatz in terms of the group-invariant variables. Through this ansatz, many explicit solutions to the nonlinear PDE are easily found, whose form would not be readily obvious just by trying simple direct ansatzes using the original independent and dependent variables in the nonlinear PDE, or by simply writing down the form for classical group-invariant solutions. In particular, some of these solutions are not invariant under any of the point symmetries of the nonlinear PDE and thus fall completely outside of classical symmetry reduction (while others coincide with explicit group-invariant solutions). Most importantly for applications, many of the solutions also have interesting analytical properties related to critical dynamics, blow-up behaviour, asymptotic behaviour and attractors.

We will illustrate this group-foliation reduction method by applying it to obtain explicit exact solutions for the class of semilinear reaction-diffusion equations

$$
u_{t}=u_{r r}+m r^{-1} u_{r}+\left(p-k u^{q}\right) u, \quad k=\mathrm{const} \neq 0, \quad p=\mathrm{const}
$$

for $u(t, r)$, with a nonlinearity power $q \neq 0,-1$, and a spatial derivative coefficient $m$. When $m$ is a positive integer, the equation (1) physically models radial diffusion with a nonlinear reaction source/sink term in $m+1$ spatial dimensions, where $r$ is the radial coordinate. When $m$ is zero, this equation similarly models reaction-diffusion in one spatial dimension, where $r$ denotes the full-line coordinate. Finally, when $m$ is not a non-negative integer, the equation (1) instead is a model for two-dimensional radial reaction-diffusion with an extra spatial-derivative reaction term $(1-m) r^{-1} u_{r}$ which can be shown [5] to add a source/sink contribution $(1-m) \lim _{r \rightarrow 0} u$ to the net flow integral $\frac{d}{d t} \int_{0}^{\infty} u r d r$ in two spatial dimensions.

Note that the constants $p, k$ in equation (1) can be freely scaled by a two-parameter transformation $t \rightarrow \lambda_{1}{ }^{2} t, r \rightarrow \lambda_{1} r, u \rightarrow \lambda_{2} u$, under which $p \rightarrow \lambda_{1}{ }^{2} p, k \rightarrow \lambda_{2}{ }^{q} k$ (with $\lambda_{1}, \lambda_{2} \neq 0$ ). Thus there is no loss of generality in assuming $k= \pm 1$ and $p=0, \pm 1$.

The symmetry structure of this class of reaction-diffusion equations (1) is given by [6]

$$
\begin{array}{ll}
\text { time translation } & \mathbf{X}_{1}=\partial / \partial t \quad \text { for all } m, q, p, \\
\text { scaling } & \mathbf{X}_{2}=2 t \partial / \partial t+r \partial / \partial r-(2 / q) u \partial / \partial u \quad \text { only for } p=0, \\
\text { space translation } & \mathbf{X}_{3}=\partial / \partial r \quad \text { only for } m=0,
\end{array}
$$

where $\mathbf{X}$ is the infinitesimal generator of a one-parameter group of point transformations acting on $(t, r, u)$. For constructing a group foliation, it is natural to use the time translation generator (2), since this is the only point symmetry admitted for all cases of the parameters $q, m$ and all values of the constants $p, k$.

In Section 2, we first set up the system of group-resolving equations given by the timetranslation symmetry (2) for the reaction-diffusion equation (1), which uses the invariants and differential invariants of the symmetry generator $\mathbf{X}_{1}$ as the independent and dependent variables in the system. We next state the form required for solutions of the group-resolving system to correspond to group-invariant solutions of the reaction-diffusion equation (1) with respect to the point symmetries generated by $\mathbf{X}_{1}, \mathbf{X}_{2}, \mathbf{X}_{3}$.

In Section 3, we explain the separation ansatz for directly reducing the system of groupresolving equations. This reduction yields an overdetermined system of differential-algebraic equations which can be readily solved by computer algebra. We present the explicit solutions of these equations and then we derive the resulting exact solutions of the reaction-diffusion equation. These solutions include explicit similarity solutions in the case $p=0$, and explicit travelling wave solutions in addition to explicit non-invariant solutions in the case $m=0$.

In Section 4, we show how the success of the reduction ansatz can be understood equivalently as constructing partially-invariant subspaces for a nonlinear operator that arises in a natural 
way from the structure of the group-resolving equations. This important observation puts our method on a wider mathematical foundation within the general theory of invariant subspaces developed by Galaktionov [7].

Finally, we make some general concluding remarks in Section 5.

\section{Group-resolving equations and symmetries}

To proceed with setting up the time-translation group foliation for the reaction-diffusion equation (1), we first write down the invariants (in terms of $t, r, u$ )

$$
x=r, \quad v=u,
$$

satisfying $\mathbf{X}_{1} x=\mathbf{X}_{1} v=0$, and the differential invariants (in terms of $u_{t}, u_{r}$ )

$$
G=u_{t}, \quad H=u_{r},
$$

satisfying $\mathbf{X}_{1}^{(1)} G=\mathbf{X}_{1}^{(1)} H=0$ where $\mathbf{X}_{1}^{(1)}$ is the first-order prolongation of the generator (2). Here $x$ and $v$ are mutually independent, while $G$ and $H$ are related by equality of mixed $r, t$ derivatives on $u_{t}$ and $u_{r}$, which gives

$$
D_{r} G=D_{t} H
$$

where $D_{r}, D_{t}$ denote total derivatives with respect to $r, t$. In addition, $v, G, H$ are related through the reaction-diffusion equation (1) by

$$
G-r^{-m} D_{r}\left(r^{m} H\right)=\left(p-k v^{q}\right) v .
$$

Now we put $G=G(x, v), H=H(x, v)$ into equations (7) and (8) and use equation (5) combined with the chain rule to arrive at a first-order PDE system

$$
\begin{aligned}
& G_{x}+H G_{v}-G H_{v}=0, \\
& G-m H / x-H_{x}-H H_{v}=\left(p-k v^{q}\right) v,
\end{aligned}
$$

with independent variables $x, v$, and dependent variables $G, H$. These PDEs are called the time-translation-group resolving system for the reaction-diffusion equation (1).

The respective solution spaces of equation (1) and system (9), (10) are related by a groupinvariant mapping that is defined through the invariants (5) and differential invariants (6).

Lemma 1. Solutions $(G(x, v), H(x, v))$ of the time-translation-group resolving system (9), (10) are in one-to-one correspondence with one-parameter families of solutions $u(t, r, c)$ of the reaction-diffusion equation (1) satisfying the translation-invariance property

$$
u(t+\epsilon, r, c)=u(t, r, \tilde{c}(\epsilon, c)),
$$

where $\tilde{c}(0, c)=c$ in terms of an arbitrary constant $c$ and parameter $\epsilon$, such that

$$
u_{t}=G(r, u), \quad u_{r}=H(r, u)
$$

constitutes a consistent pair of parametric first-order ODEs whose integration constant is $c$.

We now examine the relationship between the symmetry structure of the reaction-diffusion equation (1) and the symmetry structure inherited by the time-translation-group resolving system (9), (10). 
Firstly, through the identifications defined by the variables (5), (6), the prolongation of any point symmetry generator $\mathbf{X}=a_{1} \mathbf{X}_{1}+a_{2} \mathbf{X}_{2}+a_{3} \mathbf{X}_{3}$ of equation (1) has a natural projection to a point symmetry generator $\mathbf{Y}=a_{2} \mathbf{X}_{2}^{(1)}+a_{3} \mathbf{X}_{3}^{(1)}$ modulo $\mathbf{X}_{1}^{(1)}$ of system (9), (10). The time-translation $\mathbf{X}_{1}$ thus gets annihilated by this projection, i.e. $\mathbf{Y}_{1}=0$, while the scaling $\mathbf{X}_{2}$ and the space-translation $\mathbf{X}_{3}$ respectively project to

$$
\mathbf{Y}_{2}=x \partial / \partial x-(2 / q) v \partial / \partial v-2(1+1 / q) G \partial / \partial G-(1+2 / q) H \partial / \partial H \quad \text { when } p=0,
$$

and

$$
\mathbf{Y}_{3}=\partial / \partial x \quad \text { when } m=0 .
$$

Secondly, with respect to these inherited symmetries (12) and (13), the system (9), (10) has a reduction to ODEs yielding solutions such that $(G, H)$ is invariant respectively under scalings

$$
x \rightarrow \lambda x, \quad v \rightarrow \lambda^{-2 / q} v \quad \text { when } p=0,
$$

and under translations

$$
x \rightarrow x+\epsilon \quad \text { when } m=0 .
$$

Thus, translation-invariant solutions have the form

$$
(G, H)=(g(v), h(v))
$$

satisfying the ODE system

$$
(h / g)^{\prime}=0, \quad g-h h^{\prime}=\left(p-k v^{q}\right) v .
$$

Scaling-invariant solutions have the form

$$
(G, H)=\left(x^{-2-2 / q} g(V), x^{-1-2 / q} h(V)\right), \quad V=v x^{2 / q}
$$

satisfying the ODE system

$$
((h+2 V / q) / g)^{\prime}=-2 / g, \quad g+(1-m+2 / q) h-(h+2 V / q) h^{\prime}=-k V^{q+1} .
$$

Integration of the parametric ODEs (11) for such solutions (14), (15) and (16), (17) leads to the following two correspondence results.

Lemma 2. In the case $m=0$, there is a one-to-one correspondence between solutions of the translation-group resolving system (9), (10) with the invariant form (14) and one-parameter families of travelling-wave solutions of the reaction-diffusion equation (1) given by the groupinvariant form $u=f(\xi)$ where, modulo time-translations $t \rightarrow t+c$, the variable $\xi=r-t / a$ is an invariant of the translation symmetry $\mathbf{X}=a \partial / \partial t+\partial / \partial r=a \mathbf{X}_{1}+\mathbf{X}_{3}$ in terms of some constant a (determined by ODE system (15)).

Lemma 3. In the case $p=0$, there is a one-to-one correspondence between solutions of the translation-group resolving system (9), (10) with the invariant form (16) and one-parameter families of similarity solutions of the reaction-diffusion equation (1) given by the group-invariant form $u=r^{-2 / q} f(\xi)$ where, modulo time-translations $t \rightarrow t+c$, the variable $\xi=t / r^{2}$ is an invariant of the scaling symmetry $\mathbf{X}=2 t \partial / \partial t+r \partial / \partial r-(2 / q) u \partial / \partial u=\mathbf{X}_{2}$.

Furthermore, in all cases, static solutions $u(r)$ of the reaction-diffusion equation correspond to solutions of the translation-group resolving system with $G=0$. Hereafter we will be interested only in solutions with $G \neq 0$ and $H \neq 0$, corresponding to dynamical solutions $u(t, r)$ of the reaction-diffusion equation. 


\section{Main results}

To find explicit solutions of the group foliation system (9), (10) for $(G(x, v), H(x, v))$, we will make use of the same general homogeneity features utilized in $[4,5]$. First, the non-derivative terms $\left(p-k u^{q}\right) u$ in the reaction-diffusion equation (1) appear only as an inhomogeneous term in equation (10). Second, in both equations (9) and (10) the linear terms involve no derivatives with respect to $v$. Third, the nonlinear terms in the homogeneous equation (9) have the skewsymmetric form $H G_{v}-G H_{v}$, while the only nonlinear term appearing in the non-homogeneous equation (10) has the symmetric form $H H_{v}=\left(H^{2} / 2\right)_{v}$. Based on these features, this system can be expected to have solutions given by the separable power form

$$
G=g_{1}(x) v+g_{2}(x) v^{a}, \quad H=h_{1}(x) v+h_{2}(x) v^{a}, \quad a \neq 1 .
$$

For such a separation ansatz (18), the linear terms $G_{x}, G, H / x, H_{x}$ in equations (9) and (10) will contain the same powers $v, v^{a}$ that appear in both $G$ and $H$, and moreover the nonlinear term $H G_{v}-H_{v} G$ in the homogeneous equation (9) will produce only the power $v^{a}$ due to the identities $v^{a}(v)_{v}-\left(v^{a}\right)_{v} v=(a-1) v^{a}$ and $v(v)_{v}-(v)_{v} v=v^{a}\left(v^{a}\right)_{v}-\left(v^{a}\right)_{v} v^{a}=0$. Thus, equation (9) can be satisfied by having the coefficients of $v$ and $v^{a}$ separately vanish. Similarly the nonlinear term $H H_{v}$ in the non-homogeneous equation (10) will only yield the powers $v, v^{a}, v^{2 a-1}$. Since we have $a \neq 1$ and $q \neq 0$, equation (10) can be satisfied by again having the coefficients of $v$ and $v^{a}$ separately vanish and by also having the term containing $v^{2 a-1}$ balance the inhomogeneous term $k v^{q+1}$. In this fashion we find that equations (9) and (10) reduce to an overdetermined system of 5 algebraic-differential equations for $g_{1}(x), g_{2}(x), h_{1}(x), h_{2}(x)$, together with the relation $a=1+q / 2$. This system can be solved by a systematic integrability analysis. We have carried out this analysis using the computer algebra program CRACK [8], which contains a wide repertoire of modules for reduction of differential order and polynomial degree, splittings with respect to the independent variable(s), eliminations, substitutions, factorizations, integrations, and length-shortening of equations, among others.

Proposition 1. For $q \neq 0,-1$, the separation ansatz (18) yields altogether six solutions of the translation-group resolving system (9), (10) with $G \neq 0$ and $H \neq 0$ :

$$
\begin{aligned}
G= & \pm(3-m)\left(\frac{m-1}{m-2} k\right)^{1 / 2} v^{(m-2) /(m-1)} / x, \\
H= & \pm\left(\frac{m-1}{m-2} k\right)^{1 / 2} v^{(m-2) /(m-1)}+(1-m) v / x, \\
& m \neq 1,2, \quad q=2 /(1-m), \quad p=0, \quad k \gtrless 0 \quad((m-1)(m-2) \gtrless 0) ; \\
G= & \frac{q+4}{q+2}\left(p v+(p k)^{1 / 2} v^{1+q / 2}\right), \\
H= & \pm\left(\left(\frac{2 p}{q+2}\right)^{1 / 2} v+\left(\frac{2 k}{q+2}\right)^{1 / 2} v^{1+q / 2}\right), \\
& m=0, \quad q \gtrless-2, \quad p \gtrless 0, \quad k \gtrless 0 ; \\
G= & \frac{q+4}{q+2}\left(p v-(p k)^{1 / 2} v^{1+q / 2}\right), \quad \\
H= & \pm\left(\left(\frac{2 p}{q+2}\right)^{1 / 2} v-\left(\frac{2 k}{q+2}\right)^{1 / 2} v^{1+q / 2}\right), \\
& m=0, \quad q \gtrless-2, \quad p \gtrless 0, \quad k \gtrless 0 ; \\
G= & \frac{3}{2}\left(p v \pm(p k)^{1 / 2} \tanh \left((p / 2)^{1 / 2}\left(x+c_{1}\right)\right) v^{2}\right),
\end{aligned}
$$




$$
\begin{aligned}
H= & \pm(k / 2)^{1 / 2} v^{2}+(p / 2)^{1 / 2} \tanh \left((p / 2)^{1 / 2}\left(x+c_{1}\right)\right) v \\
& m=0, \quad q=2, \quad p>0, \quad k>0 \\
G= & \frac{3}{2}\left(p v \mp(-p k)^{1 / 2} \tan \left((-p / 2)^{1 / 2}\left(x+c_{1}\right)\right) v^{2}\right), \\
H= & \pm(k / 2)^{1 / 2} v^{2}-(-p / 2)^{1 / 2} \tan \left((-p / 2)^{1 / 2}\left(x+c_{1}\right)\right) v \\
& m=0, \quad q=2, \quad p<0, \quad k>0 \\
G= & \pm 3(k / 2)^{1 / 2} v^{2} /\left(x+c_{1}\right), \\
H= & \pm(k / 2)^{1 / 2} v^{2}+v /\left(x+c_{1}\right), \\
& m=0, \quad q=2, \quad p=0, \quad k>0 .
\end{aligned}
$$

Solution (19) satisfies the scaling-invariance reduction (16); solutions (20) and (21) satisfy the translation-invariance reduction (14); solution (24) for $c_{1}=0$ satisfies the scaling-invariance reduction (16).

Remark 1. A shift of the arbitrary constant $c_{1} \rightarrow c_{1} \pm i \pi /(2 p)^{1 / 2}$ in solution (22) and $c_{1} \rightarrow$ $c_{1} \pm \pi /(-2 p)^{1 / 2}$ in solution (23) respectively yields the two further solutions

$$
\begin{aligned}
G= & \frac{3}{2}\left(p v \pm(p k)^{1 / 2} \operatorname{coth}\left((p / 2)^{1 / 2}\left(x+c_{1}\right)\right) v^{2}\right) \\
H= & \pm(k / 2)^{1 / 2} v^{2}+(p / 2)^{1 / 2} \operatorname{coth}\left((p / 2)^{1 / 2}\left(x+c_{1}\right)\right) v \\
& m=0, \quad q=2, \quad p>0, \quad k>0
\end{aligned}
$$

and

$$
\begin{aligned}
G= & \frac{3}{2}\left(p v \pm(-p k)^{1 / 2} \cot \left((-p / 2)^{1 / 2}\left(x+c_{1}\right)\right) v^{2}\right), \\
H= & \pm(k / 2)^{1 / 2} v^{2}+(-p / 2)^{1 / 2} \cot \left((-p / 2)^{1 / 2}\left(x+c_{1}\right)\right) v, \\
& m=0, \quad q=2, \quad p<0, \quad k>0,
\end{aligned}
$$

as obtained through the trigonometric identities

$$
\tanh (\theta \pm i \pi / 2)=\operatorname{coth}(\theta), \quad \tan (\theta \pm \pi / 2)=-\cot (\theta) .
$$

The form of the separation ansatz (18) can be naturally generalized to include additional powers

$$
G=g_{1}(x) v+\sum_{i=1}^{N} g_{1+i}(x) v^{a_{i}}, \quad H=h_{1}(x) v+\sum_{i=1}^{N} h_{1+i}(x) v^{a_{i}},
$$

where

$$
a_{i} \neq 1, \quad a_{i}-a_{j} \neq 0 .
$$

This multi-term ansatz (for $N \geq 2$ ) leads to a considerably more complicated analysis compared to the previous two-term ansatz (with $N=1$ ). Specifically, the homogeneous equation (9) now contains the powers $v^{a_{i}+a_{j}-1}$ in addition to $v, v^{a_{i}}$, while the non-homogeneous equation (10) contains the further powers $v^{2 a_{i}-1}$, and $v^{q+1}$. To determine the exponents in these powers, a systematic examination of the possible balances (which rapidly increase in number with $N$ ) is necessary. We carry out this analysis by computer algebra, again using the program CRACK [8]. In particular, for any fixed $N \geq 1$, CRACK can be run automatically by setting up a priority list of modules to be tried recursively with case splittings given a high priority, in order to split 
equations (9) and (10) under the ansatz (25), (26) and in each case solve the resulting overdetermined system of algebraic-differential equations for $g_{i}$ and $h_{i}, i=1,2, \ldots, N$. Importantly, the modules in CRACK are able to organize the case distinctions in an efficient way that lessens a combinatorial explosion by using algebraic conditions (equalities and inequalities) arising in the steps of solving the equations to keep track of whether a possible balance of two powers of $v$ generates a new case (or subcase) which has to be solved or not. Running CRACK, we find that the system (9), (10), (25), (26) has solutions when $N=2$ but not when $N=3$. (Based on this outcome we have not tried to investigate the system when $N>3$.)

Proposition 2. For $q \neq 0,-1$, and $N \leq 3$, the separation ansatz (25) yields four additional solutions of the translation-group resolving system (9), (10) with $G \neq 0$ :

$$
\begin{aligned}
G= & 3 p\left(v+(k / p)^{2 / 3} v^{1 / 2}+(k / p)^{1 / 3}\right), \\
H= & \pm(2 p)^{1 / 2}\left(v+(k / p)^{2 / 3} v^{1 / 2}+(k / p)^{1 / 3}\right), \\
& m=0, \quad q=-3 / 2, \quad p>0, \quad k \gtrless 0 ; \\
G= & 4 p\left(v+(-4 k / p)^{1 / 4} v^{1 / 3}+(-k / p)^{1 / 2} v^{-1 / 3}\right), \\
H= & \pm(3 p)^{1 / 2}\left(v+(-4 k / p)^{1 / 4} v^{1 / 3}+(-k / p)^{1 / 2} v^{-1 / 3}\right), \\
& m=0, \quad q=-8 / 3, \quad p>0, \quad k<0 ; \\
G= & 4 p\left(v-(-4 k / p)^{1 / 4} v^{1 / 3}+(-k / p)^{1 / 2} v^{-1 / 3}\right), \\
H= & \pm(3 p)^{1 / 2}\left(v-(-4 k / p)^{1 / 4} v^{1 / 3}+(-k / p)^{1 / 2} v^{-1 / 3}\right), \\
& m=0, \quad q=-8 / 3, \quad p>0, \quad k<0 ; \\
G= & \pm 3(-k / 8)^{1 / 2}\left(v \pm(-2 k)^{-1 / 2} x^{-1}\right)^{2} / x, \\
H= & \pm(-k / 2)^{1 / 2}\left(v^{2}+3 /(2 k) x^{-2}\right), \\
& m=3 / 2, \quad q=2, \quad p=0, \quad k<0 .
\end{aligned}
$$

Solutions (27), (28), (29) satisfy the translation-invariance reduction (14); solution (30) satisfies the scaling-invariance reduction (16).

We now obtain explicit solutions $u(t, r)$ of the reaction-diffusion equation (1) from the solutions $(G(x, v), H(x, v))$ of its translation-group resolving system (9), (10) by integrating the corresponding pair of parametric first-order ODEs (11). This integration yields a one-parameter solution family $u(t, r, c)$ which is closed under the action of the group of time-translations $t \rightarrow t+\epsilon$.

Theorem 1. The semilinear reaction-diffusion equation (1) has the following exact solutions arising from the explicit solutions of its translation-group resolving system found in Propositions 1 and 2 (and Remark 1):

$$
\begin{aligned}
u= & \left( \pm\left(\frac{k}{(m-2)(m-1)}\right)^{1 / 2}\left(\frac{r}{2}-\frac{(m-3)(t+c)}{r}\right)\right)^{m-1}, \\
& m \neq 1,2, \quad q=2 /(1-m), \quad p=0, \quad k \gtrless 0 \quad((m-1)(m-2) \gtrless 0) ; \quad(31) \\
u= & \left(-(k / p)^{1 / 2}+\exp \left(\mp q\left(\frac{p}{2(q+2)}\right)^{1 / 2}\left(r \pm(q+4)\left(\frac{p}{2(q+2)}\right)^{1 / 2}(t+c)\right)\right)\right)^{-2 / q}, \\
& m=0, \quad q \gtrless-2, \quad p \gtrless 0, \quad k \gtrless 0 ; \\
u=\left((k / p)^{1 / 2}+\exp \left(\mp q\left(\frac{p}{2(q+2)}\right)^{1 / 2}\left(r \pm(q+4)\left(\frac{p}{2(q+2)}\right)^{1 / 2}(t+c)\right)\right)\right)^{-2 / q}, & \\
& m=0, \quad q \gtrless-2, \quad p \gtrless 0, \quad k \gtrless 0 ;
\end{aligned}
$$




$$
\begin{aligned}
& u=\frac{\cosh \left((p / 2)^{1 / 2}\left(r+c_{1}\right)\right)}{(k / p)^{1 / 2} \sinh \left((p / 2)^{1 / 2}\left(r+c_{1}\right)\right) \pm \exp (-3 p(t+c) / 2)}, \\
& m=0, \quad q=2, \quad p>0, \quad k>0 \text {; } \\
& u=\frac{\sinh \left((p / 2)^{1 / 2}\left(r+c_{1}\right)\right)}{(k / p)^{1 / 2} \cosh \left((p / 2)^{1 / 2}\left(r+c_{1}\right)\right) \pm \exp (-3 p(t+c) / 2)}, \\
& m=0, \quad q=2, \quad p>0, \quad k>0 \\
& u=\frac{\cos \left((-p / 2)^{1 / 2}\left(r+c_{1}\right)\right)}{(-k / p)^{1 / 2} \sin \left((-p / 2)^{1 / 2}\left(r+c_{1}\right)\right) \pm \exp (-3 p(t+c) / 2)}, \\
& m=0, \quad q=2, \quad p<0, \quad k>0 \\
& u=\frac{\sin \left((-p / 2)^{1 / 2}\left(r+c_{1}\right)\right)}{(-k / p)^{1 / 2} \cos \left((-p / 2)^{1 / 2}\left(r+c_{1}\right)\right) \mp \exp (-3 p(t+c) / 2)}, \\
& m=0, \quad q=2, \quad p<0, \quad k>0 \text {; } \\
& u=\left( \pm(k / 2)^{1 / 2}\left(\frac{r+c_{1}}{2}-\frac{3(t+c)}{r+c_{1}}\right)\right)^{-1} \text {, } \\
& m=0, \quad q=2, \quad p=0, \quad k>0 \text {; } \\
& u=\frac{t+c-r^{2}}{\sqrt{2 k}\left(t+c+r^{2} / 3\right) r}, \\
& m=3 / 2, \quad q=2, \quad p=0, \quad k>0 \text {; } \\
& u=(k /(8 p))^{2 / 3}(\sqrt{3} \cot \phi(\xi)-1)^{2} \quad \text { where } \quad \xi=r \pm 3(p / 2)^{1 / 2}(t+c), \\
& \exp (-\phi(\xi) / \sqrt{3}) \sin \phi(\xi)=\sqrt{3}(k /(8 p))^{1 / 3} \exp \left((2 p)^{1 / 2} \xi\right), \\
& m=0, \quad q=-3 / 2, \quad p>0, \quad k \gtrless 0 ; \\
& u=(-k /(4 p))^{3 / 8}(\cot \phi(\xi)-1)^{3 / 2} \quad \text { where } \xi=r \pm 4(p / 3)^{1 / 2}(t+c) \text {, } \\
& \exp (-2 \phi(\xi)) \sin \phi(\xi)=(-k /(4 p))^{1 / 4} \exp \left(2(p / 3)^{1 / 2} \xi\right), \\
& m=0, \quad q=-8 / 3, \quad p>0, \quad k<0 ; \\
& u=(-k /(4 p))^{3 / 8}(\cot \phi(\xi)+1)^{3 / 2} \quad \text { where } \xi=r \pm 4(p / 3)^{1 / 2}(t+c) \text {, } \\
& \exp (2 \phi(\xi)) \sin \phi(\xi)=(-k /(4 p))^{1 / 4} \exp \left(2(p / 3)^{1 / 2} \xi\right), \\
& m=0, \quad q=-8 / 3, \quad p>0, \quad k<0,
\end{aligned}
$$

where $c, c_{1}$ are arbitrary constants.

Remark 2. Solutions (34) and (35) are related by shifts of the arbitrary constants $c_{1} \rightarrow c_{1} \pm$ $i \pi /(2 p)^{1 / 2}$ and $c \rightarrow c \mp i \pi /(3 p)$ through the trigonometric identities

$$
\sinh (\theta \pm i \pi / 2)= \pm i \cosh (\theta), \quad \cosh (\theta \pm i \pi / 2)= \pm i \sinh (\theta)
$$

while solutions (36) and (37) are related in a similar way through the trigonometric identities

$$
\sin (\theta \pm \pi / 2)= \pm \cos (\theta), \quad \cos (\theta \pm \pi / 2)=\mp \sin (\theta)
$$

Modulo time-translations, solutions (31) and (39) are similarity solutions, since they have the form shown in Lemma 3. These solutions have been obtained in our previous work [5] using a two-term separation ansatz to solve the group-resolving equations given by the scaling symmetry (3) for the reaction-diffusion equation (1) in the case $p=0$. Solution (38) can be obtained from the $m=0$ case of solution (31) after a space-translation is applied. 
Solutions (32), (33), (40), (41) and (42) are travelling-wave solutions with the form shown in Lemma 2 (where the constant $a$ is the wave speed). The two solutions (32) and (33) were first obtained in [11] through standard symmetry reduction. To the best knowledge of the authors, the other three solutions (40), (41) and (42) are new.

In contrast, solutions (34) to (37) are non-invariant solutions, as they do not have the form of travelling waves whereas the symmetry group of the reaction-diffusion equation (1) in the case $p \neq 0$ and $m=0$ is generated entirely by time-translation and space-translation symmetries (2) and (4). These solutions have been found previously in [9, 10] using Bluman and Cole's nonclassical method.

\section{Invariance properties of the reduction ansatz}

As we will now explain, the success of the separation ansatzes (18) and (25) for reducing the system of group-foliation equations (9) and (10) has a mathematically natural interpretation within the theory of invariant subspaces [7].

We start by writing the group-foliation equations in an evolutionary operator form

$$
\left(\begin{array}{l}
G \\
H
\end{array}\right)_{x}=\Phi\left(\left(\begin{array}{l}
G \\
H
\end{array}\right)\right)=\left(\begin{array}{c}
0 \\
\left(k v^{q}-p\right) v
\end{array}\right)+\left(\begin{array}{c}
0 \\
G-m H / x
\end{array}\right)+\left(\begin{array}{c}
G H_{v}-H G_{v} \\
-H H_{v}
\end{array}\right)
$$

where $\Phi$ defines a nonlinear operator acting on the pair of variables $(G, H)$. Then we view the ansatz (18) as defining a linear space of functions with a separable power form

$$
\left(\begin{array}{l}
G \\
H
\end{array}\right)=\left(\begin{array}{l}
g_{1} \\
h_{1}
\end{array}\right) v+\left(\begin{array}{l}
g_{2} \\
h_{2}
\end{array}\right) v^{a}, \quad a \neq 1
$$

where the coefficients depend only on $x$. This linear space (44) is not invariant under $\Phi$ because

$$
\begin{aligned}
\Phi\left(\left(\begin{array}{l}
g_{1} \\
h_{1}
\end{array}\right) v+\left(\begin{array}{l}
g_{2} \\
h_{2}
\end{array}\right) v^{a}\right) \\
=\left(\begin{array}{c}
0 \\
g_{1}-m h_{1} / x-h_{1}^{2}-p
\end{array}\right) v+\left(\begin{array}{c}
(a-1)\left(h_{2} g_{1}-h_{1} g_{2}\right) \\
g_{2}-m h_{2} / x-(a+1) h_{1} h_{2}
\end{array}\right) v^{a} \\
\quad+\left(\begin{array}{c}
0 \\
-a h_{2}^{2}
\end{array}\right) v^{2 a-1}+\left(\begin{array}{l}
0 \\
k
\end{array}\right) v^{q+1}
\end{aligned}
$$

produces terms that have additional powers $v^{2 a-1}, v^{q+1}$.

However, the operator $\Phi$ will preserve a set of functions contained in the linear space (44) if the power $a$ and the coefficients $g_{1}, g_{2}, h_{1}, h_{2}$ satisfy the conditions

$$
2 a-1=q+1, \quad a h_{2}^{2}=k,
$$

so that the terms containing $v^{2 a-1}$ and $v^{q+1}$ cancel out. In this sense the linear space will then be partially-invariant [7] under $\Phi$. As a consequence of this invariance property, the evolution equation (43) for $(G, H)$ reduces to an overdetermined system of differential-algebraic equations which we can solve for $g_{1}, g_{2}, h_{1}$, as discussed in Section 2 .

The separable power ansatz (18) thus can be viewed as explicitly constructing a partiallyinvariant linear subspace for the group-foliation operator $\Phi$. A similar discussion applies to the multi-term ansatz (25).

It is worth pointing out that apart from the mathematical interpretation given to the ansatzes (18) and (25), the theory of (partially) invariant linear subspaces does not provide any general constructive method or algorithm for finding a successful ansatz. So the main aspect of 
the present work is to demonstrate the effectiveness of a general separation ansatz for solving group-foliation equations. We also emphasize that this approach is much more effective than if an analogous separation method were to be applied either directly for solving the given reactiondiffusion PDE or instead for seeking a (partially) invariant linear subspace for that PDE itself.

\section{Concluding remarks}

In general the method of group foliation reduction using a separation ansatz as illustrated in this paper is able to yield exact solutions to nonlinear 2nd order PDEs with power nonlinearities.

This method works with any admitted group of point (or contact) symmetries and gives a systematic reduction of the group foliation equations into an overdetermined system consisting of algebraic equations and 1st order differential equations that can be derived and in most cases solved by means of computer algebra (e.g. using the program CRACK [8]). In particular, for a given nonlinear 2nd order PDE having two independent variables, solutions are produced in an explicit form, whereas standard symmetry reduction only gives a 2nd order ODE that still has to be solved to find group-invariant solutions explicitly and in general this step can be quite difficult.

Moreover, because the group foliation equations contain all solutions of the given nonlinear PDE, the method can yield solutions that are not invariant under any of the point (or contact) symmetries admitted by the nonlinear PDE.

It is straightforward to extend this method to higher-order PDEs with power nonlinearities. As well, it should be possible to apply the same method to PDEs having more general forms of nonlinearities by utilizing a general separation of variables ansatz with respect to all of the independent variables in the group foliation equations. In general, the success of such an ansatz can be interpreted mathematically [7] as constructing a (partially) invariant linear subspace for a nonlinear operator coming from the structure of the group foliation equations.

Related work using a similar group-foliation method applied to nonlinear diffusion equations appears in [12]. Group foliation equations were first used successfully in [13, 14, 15, 16] for obtaining exact solutions to nonlinear PDEs by a different method that is applicable when the group of point symmetries of a given PDE is infinite-dimensional, compared to the example of a finite-dimensional symmetry group considered both in $[4,5]$ and in the present work.

\section{Acknowledgements}

S. Anco and T. Wolf are each supported by an NSERC research grant. S. Ali thanks the Mathematics Department of Brock University for support during the period of a research visit when this paper was written. Computations were partly performed on computers of the Sharcnet consortium (www.sharcnet.ca). The referees and the editor are thanked for valuable comments which have improved this paper.

\section{References}

[1] Ovsiannikov L.V., Group analysis of differential equations, Academic Press, Inc., New York - London, 1982.

[2] Olver P.J., Applications of Lie groups to differential equations, 2nd ed., Graduate Texts in Mathematics, Vol. 107, Springer-Verlag, New York, 1993.

[3] Bluman G., Anco S.C., Symmetry and integration methods for differential equations, Applied Mathematical Sciences, Vol. 154, Springer-Verlag, New York, 2002.

[4] Anco S.C., Liu S., Exact solutions of semilinear radial wave equations in $n$ dimensions, J. Math. Anal. Appl. 297 (2004), 317-342, math-ph/0309049. 
[5] Anco S.C., Ali S., Wolf T., Symmetry analysis and exact solutions of semilinear heat flow in multidimensions, J. Math. Anal. Appl. 379 (2011), 748-763, arXiv:1011.4633.

[6] Dorodnitsyn V.A., On invariant solutions of the equation of nonlinear heat conduction with a source, USSR Comp. Math. Math. Phys. 22 (1982), 115-122.

[7] Galaktionov V.A., Svirshchevskii S.R., Exact solutions and invariant subspaces of nonlinear partial differential equations in mechanics and physics, Chapman \& Hall/CRC, Boca Raton, 2007.

[8] Wolf T., Applications of CRACK in the classification of integrable systems, in Superintegrability in Classical and Quantum Systems, CRM Proc. Lecture Notes, Vol. 37, Amer. Math. Soc., Providence, RI, 2004, 283-300, http://lie.math. brocku.ca/crack/demo/.

[9] Clarkson P.A., Mansfield E.L., Symmetry reductions and exact solutions of a class of nonlinear heat equations, Phys. D 70 (1993), 250-288, solv-int/9306002.

[10] Arrigo D.J., Hill J.M., Broadbridge P., Nonclassical symmetry reductions of the linear diffusion equation with a nonlinear source, IMA J. Appl. Math. 52 (1994), 1-24.

[11] Vijayakumar K., On the integrability and exact solutions of the nonlinear diffusion equation with a nonlinear source, J. Austral. Math. Soc. Ser. B 39 (1998), 513-517.

[12] Qu C., Zhang S.-L., Group foliation method and functional separation of variables to nonlinear diffusion equations, Chinese Phys. Lett. 22 (2005), 1563-1566.

[13] Golovin S.V., Applications of the differential invariants of infinite dimensional groups in hydrodynamics, Commun. Nonlinear Sci. Numer. Simul. 9 (2004), 35-51.

[14] Nutku Y., Sheftel M.B., Differential invariants and group foliation for the complex Monge-Ampère equation, J. Phys. A: Math. Gen. 34 (2001), 137-156.

[15] Martina L., Sheftel M.B., Winternitz P., Group foliation and non-invariant solutions of the heavenly equation, J. Phys. A: Math. Gen. 34 (2001), 9243-9263, math-ph/0108004.

[16] Sheftel M.B., Method of group foliation and non-invariant solutions of partial differential equations. Example: the heavenly equation, Eur. Phys. J. B Condens. Matter Phys. 29 (2002), 203-206. 